\title{
COVID-19 in Severe Asthma Network in Italy (SANI) patients: Clinical features, impact of comorbidities and treatments
}

To the Editor,

Since the end of February 2020, Italy, first non-Asian Country, has reported an ever increasing number of COronaVIrus Disease 19 (COVID-19) patients, which has reached over 200000 confirmed severe acute respiratory syndrome coronavirus-2 (SARS-CoV-2) infected subjects, resulting in more than 34000 deaths (data updated to June 19 th, $2020^{1}$ ).

Patients with asthma are potentially more severely affected by SARS-CoV-2 infection and respiratory viruses are known to be associated with severe adverse asthma outcomes, including increased risk of asthma exacerbation episodes. ${ }^{2}$ Nonetheless, according to the epidemiological studies published so far, asthma is not among the most common clinical conditions in COVID-19 patients. ${ }^{3}$

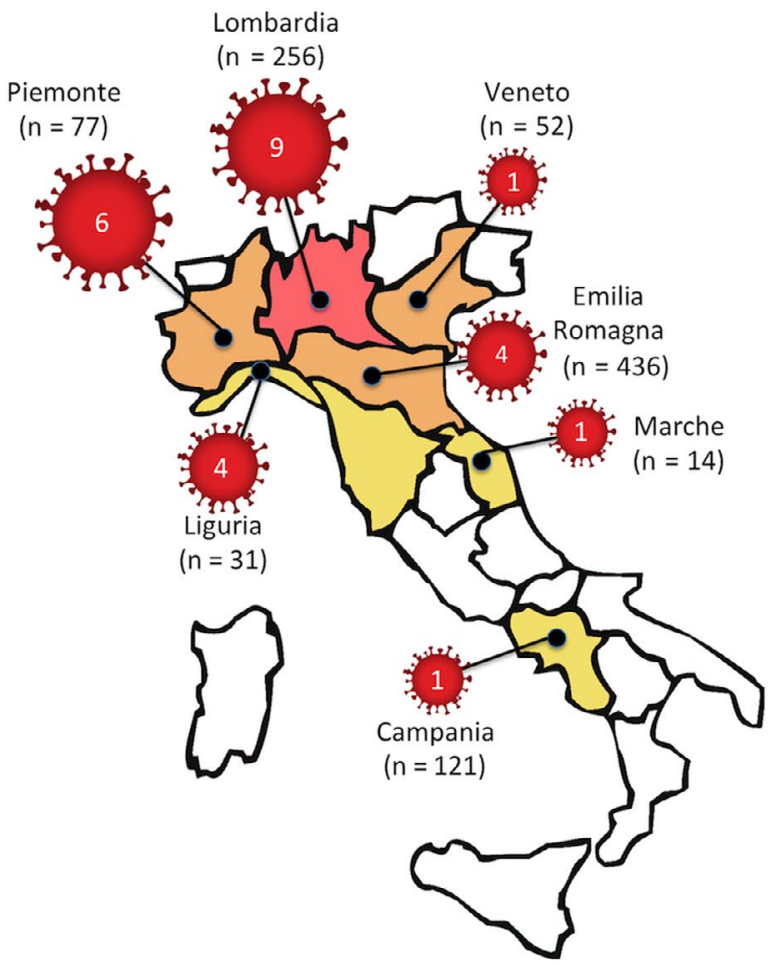

About $5 \%-10 \%$ of asthmatics are severe, ${ }^{4}$ and one would expect increased vulnerability to SARS-CoV-2 infection, but no data are so far available to confirm this hypothesis.

We investigated the incidence of COVID-19, describing its clinical course, in the population of the Severe Asthma Network in Italy (SANI), one of the largest registry for severe asthma worldwide, ${ }^{5}$ and in an additional Center (Azienda Ospedaliero Univeristaria di Ferrara, Ferrara, Italy). All centres have been contacted and inquired to report confirmed or highly suspect cases of COVID-19 (ie, patients with symptoms, laboratory findings and lung imaging typical of COVID-19 but without access to nasopharyngeal or oropharyngeal swab specimens because of clinical contingencies/emergency) among their cohorts of severe asthma. Demographic and clinical have been obtained from the
SEVERE ASTHMATICS WITH COVID-19

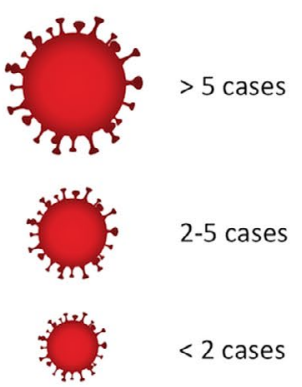

SUBJECTS WITH POSITIVE NASOPHARYNGEAL SWAB FOR SARS-COV-2

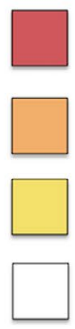

$>30000$ positive subjects 
TAB LE 1 Demographic and clinical characteristics of severe asthmatics with COVID-19

\begin{tabular}{|c|c|c|c|c|c|c|c|c|}
\hline ID & Region & $\begin{array}{l}\text { Suspect or Confirmed } \\
\text { COVID-19 }\end{array}$ & Age & Sex & BMI & Atopy & Smoker & Comorbidities \\
\hline 1 & Emilia Romagna & Confirmed & 48 & $\mathrm{~F}$ & 34 & Yes & No & GERD \\
\hline 3 & Emilia Romagna & Confirmed & 65 & $\mathrm{~F}$ & 33 & Yes & No & BX, CVD, Anxiety, Osteoporosis \\
\hline 4 & Emilia Romagna & Suspect & 32 & M & 33 & Yes & No & AR \\
\hline 5 & Lombardia & Confirmed & 45 & $\mathrm{~F}$ & 20 & Yes & Ex & CRSwNP, GERD, \\
\hline 6 & Lombardia & Confirmed & 45 & $\mathrm{~F}$ & 27 & No & No & CRSWNP, GERD \\
\hline 10 & Lombardia & Confirmed & 62 & M & 33 & Yes & No & AR, CRSsNP, GERD, BX, HTN \\
\hline 11 & Lombardia & Confirmed & 66 & $\mathrm{~F}$ & 28 & Yes & Yes & $\begin{array}{l}\text { AR, CRSsNP, CVD, Glaucoma, } \\
\text { Cataract, NIDDM }\end{array}$ \\
\hline 12 & Lombardia & Suspect & 51 & $\mathrm{~F}$ & 25 & Yes & No & None \\
\hline 13 & Lombardia & Suspect & 37 & $\mathrm{~F}$ & 19 & No & No & CRSWNP, AD \\
\hline 14 & Piemonte & Suspect & 66 & $\mathrm{~F}$ & 23 & Yes & No & AR, CRSwNP, GERD \\
\hline 18 & Piemonte & Suspect & 61 & M & 25 & No & No & CRSWNP \\
\hline 19 & Piemonte & Suspect & 55 & $\mathrm{~F}$ & 23 & Yes & No & None \\
\hline 20 & Veneto & Confirmed & 53 & $\mathrm{~F}$ & 23 & No & No & None \\
\hline 21 & Liguria & Suspect & 50 & M & 28 & Yes & Yes & AR, CRSwNP \\
\hline 22 & Liguria & Suspect & 46 & $\mathrm{~F}$ & 27 & Yes & Yes & None \\
\hline 23 & Liguria & Suspect & 70 & M & 25 & No & Ex & CRSwNP, Osteopororis \\
\hline 24 & Liguria & Suspect & 60 & $\mathrm{~F}$ & 20 & No & No & CRSWNP, BX \\
\hline 25 & Campania & Confirmed & 70 & $\mathrm{~F}$ & 39 & Yes & Ex & AR, GERD, CVD, NIDDM \\
\hline 26 & Marche & Confirmed & 51 & M & 28 & No & No & CRSwNP \\
\hline
\end{tabular}

Abbreviations: AD, atopic dermatitis; ALB, albuterol; AMC, amoxicillin/clavulanate; AR, allergic rhinitis; AZM, azithromycin; BENRA, benralizumab; $\mathrm{BX}$, bronchiectasis; Cax, ceftriaxone; CIP, ciprofloxacin; CRSsNP, chronic rhinosinusits without nasal polyps; CRSwNP, chronic rhinosinusitis with nasal polyps; CVD, cardiovascular diseases; GERD, gastroesophageal reflux disease; HCQ, hydroxychloroquine; HTN, hypertension; IBP, ibuprofen; ICS/LABA, Inhaled corticosteroids/Long-acting beta2-agonists; LAMA, long-acting muscarinic agents; LMWH, low molecular weight heparins; LPV/r, lopinavir/ritonavir; LTRA, leukotriene receptor antagonists; LVX, levofloxacin; MDD, major depressive disorder; MEPO, mepolizumab; MV, mechanical ventilation; NIDDM, noninsulin-dependent diabetes mellitus; NIV, noninvasive ventilation; OCS, oral corticosteroids; OMA, omalizumab; PCM, paracetamol; TMP-SMX, trimethoprim/sulfamethoxazole; TOZ, tocilizumab. 


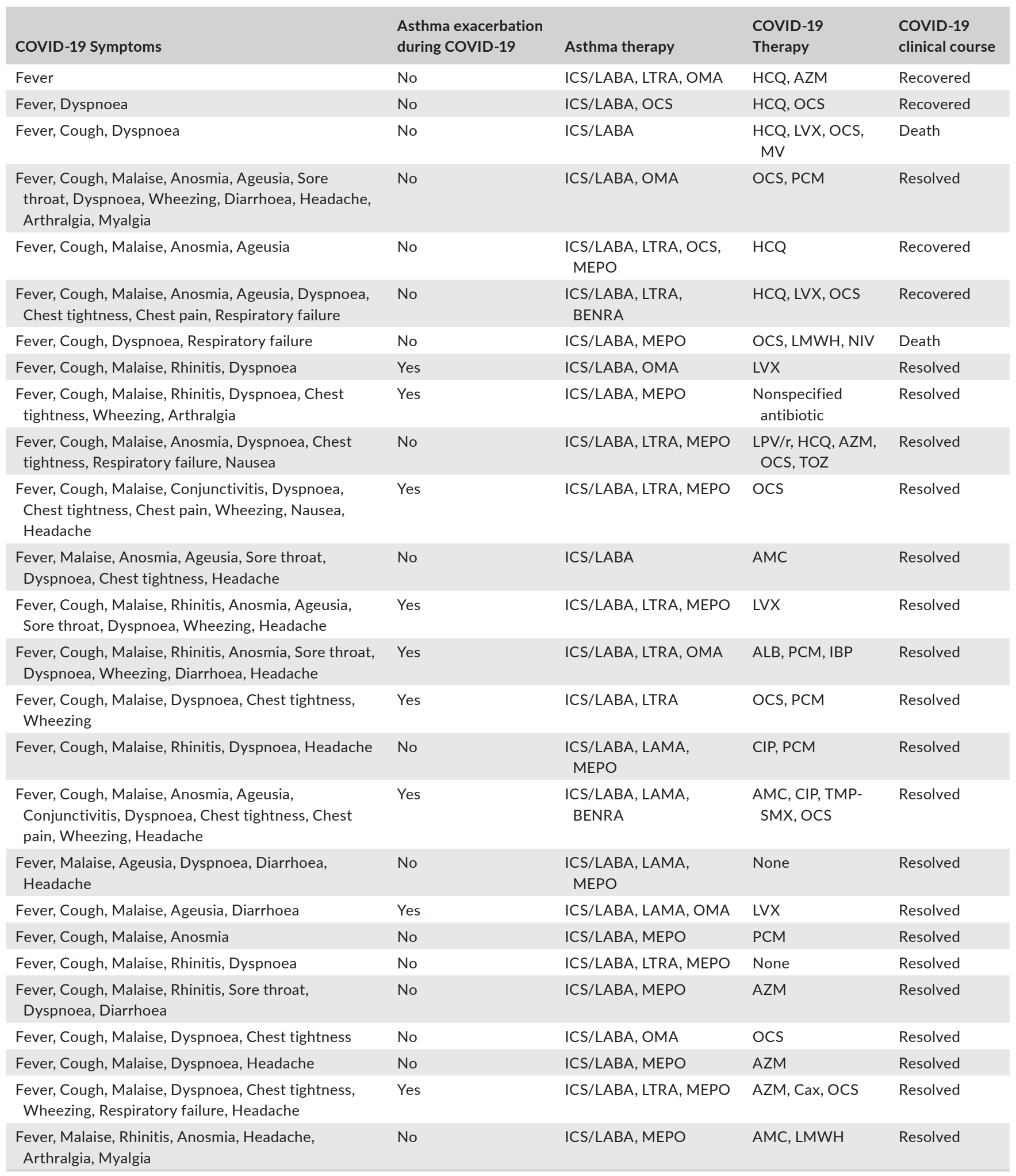


registry platform and collected from the additional Center. Additional data about COVID-19 symptoms, treatment and clinical course have been collected for all cases reported.

Ethical issues and statistical analysis are reported in the Appendix S1.

The entire severe asthmatics population accounted for 1504 patients, $65 \%$ of them were treated with biologicals (anti-IL5 or anti-IL5R agents: 52.9\%, anti-IgE: 47.1\%). Twenty-six (1.73\%) patients had confirmed (11) or highly suspect COVID-19 (15); eighteen (69.2\%) were females, and mean age was $56.2 \pm 10$ years. The geographical distribution of COVID-19 cases is presented in Figure 1.

Nine (34.6\%) infected patients experienced worsening of asthma during the COVID-19 symptomatic period; four of them needed a short course of oral corticosteroids for controlling asthma exacerbation symptoms.

The most frequent COVID-19 symptoms were fever (100\% of patients), malaise (84.6\%), cough (80.8\%), dyspnoea (80.8\%), headache (42.3\%) and loss of smell (42.3\%). Four patients (15.3\%) have been hospitalized, one of which in intensive care unit; among hospitalized patients, two (7.7\%) died for COVID-19 interstitial pneumonia (no deaths among the nonhospitalized patients).

Severe asthmatics, affected by COVID-19, had a significantly higher prevalence of noninsulin-dependent diabetes mellitus (NIDDM) compared to noninfected severe asthma patients (15.4\% vs $3.8 \%, P=.002$; odds ratio: 4.7$)$. No difference was found in other comorbidities; however, patients with severe asthma and NIDDM had a not statistically significant trend of higher BMI (31.9 vs 26.9 $P=.09$ ), suggesting a possible interaction between obesity and NIDDM as risk factors for COVID-19 in severe asthmatics.

Twenty-one patients with COVID-19 were on biologicals: 15 (71\%) on anti-IL-5 or anti-IL5R agents (Mepolizumab $n=13$; Benralizumab $n=2$ - counting for the $2.9 \%$ of all severe asthmatics treated with anti-IL5 in our study population) and 6 (29\%) on anti-IgE (Omalizumab - 1.3\% of all severe asthmatics treated with omalizumab in our study population).

Table 1 describes the 26 COVID-19 patients.

In conclusion, in our large cohort of severe asthmatics, COVID19 was infrequent, not supporting the concept of asthma as a particularly susceptible condition to SARS-CoV2 infection. This is in line with the under-reported asthma cases among patients with COVID19 patients. $^{3}$ The COVID-19 related mortality rate in our cohort of patients was $7.7 \%$, lower than in the general population $(14.5 \%$ in $\left(\right.$ taly $\left.^{1}\right)$. These findings suggest that severe asthmatics are not at high risk of SARS-CoV-2 infection and of severe forms of COVID19. There are potentially different reasons for this. Self-containment is the first, because of the awareness of viruses acting as a trigger for exacerbations, and therefore, they could have acted with greater caution, scrupulously respecting social distancing, lockdown and hygiene rules of prevention, and being more careful in regularly taking asthma medications.

Another possible explanation stands in the intrinsic features of type- 2 inflammation that characterizes a great proportion of severe asthmatics. Respiratory allergies and allergen exposures are associated with significant reduction in angiotensin-converting enzyme 2 (ACE2) expression, ${ }^{6}$ the cellular receptor for SARSCoV-2. Interestingly, ACE2 and transmembrane serine protease 2 (TMPRSS2) (another protein mediating SARS-CoV-2 cell entry) have been found highly expressed in asthmatics with concomitant NIDDM, ${ }^{7}$ the only comorbidity that was more frequent reported in our COVID-19 severe asthmatics.

The third possible explanation refers to the possibility that inhaled corticosteroids (ICS) might prevent or mitigate the development of Coronaviruses infections. Severe asthmatics, treated with high doses of ICS, ${ }^{4}$ may have been protected from SARS-CoV-2 infection.

Noteworthy, among our case series of severe asthmatics with COVID-19, the proportion of those treated anti-IL5 biologics was higher (71\%) compared to those treated with anti-IgE (29\%). Although the number of cases is too small to draw any conclusion, it is tempting to speculate that different biological treatments can have specific and different impact on antiviral immune response, as suggested for anti-IgE as protective for other viral infections. ${ }^{8}$ Moreover, we may speculate of the consequence of blood eosinophils reduction induced by anti-IL5 agents, as more than $70 \%$ of infected patients were treated with them: Eosinopenia has been reported in 52\%-90\% of COVID-19 patients worldwide, and it has been suggested as a risk factor for more severe COVID-19. ${ }^{9}$ So far, no other large series of severe asthmatics treated with biologicals infected by COVID-19 has been published, so our speculations on the role of biologicals in modulating the risk of COVID-19 need further evidence.

In conclusion, in our large cohort of severe asthmatics, only a small minority experienced symptoms consistent with COVID-19, and these patients had peculiar clinical features including high prevalence of NIDDM as comorbidity. Further real-life registry-based studies are needed to confirm our findings and to extend the evidence that severe asthmatics are at low risk of developing COVID-19.

\section{ACKNOWLEDGMENTS}

We are grateful to Silvia Rabotti, Concetta Sirena \& the SANI staff for the invaluable work in managing the network.

\section{CONFLICT OF INTEREST}

Enrico Heffler reports participation to advisory boards and personal fees from AstraZeneca, Sanofi, GSK, Novartis, Circassia, Nestlè Purina, Boheringer Ingheleim, Valeas, outside the submitted work. Aikaterini Detoraki does not have any conflict of interest to report. Marco Contoli reports grants from Chiesi, University of Ferrara-Italy, personal fees from Chiesi, AstraZeneca, Boehringer Ingelheim, Alk-Abello, GlaxoSmithKline, Novartis, Zambon, outside the submitted work. Alberto Papi reports grants,, personal fees and non-financial support from AstraZeneca, Menarini, grants, personal fees, nonfinancial support and other from Boehringer Ingelheim, Chiesi Farmaceutici, TEVA, personal fees, nonfinancial 
support and other from GlaxoSmithKline, Mundipharma, Zambon, Novartis, Sanofi/Regeneron, personal fees from Roche, Edmondpharma, grants from Fondazione Maugeri, Fondazione Chiesi, outside the submitted work. Giovanni Paoletti does not have any conflict of interest to report. Giacomo Malipiero does not have any conflict of interest to report. Luisa Brussino does not have any conflict of interest to report. Claudia Crimi reports personal fees from Menarini. Daniela Morrone does not have any conflict of interest to report. Marianna Padovani does not have any conflict of interest to report. Giuseppe Guida does not have any conflict of interest to report. Alberto Giovanni Gerli does not have any conflict of interest to report. Stefano Centanni reports personal fees from GLAXOSMITHKLINE, NOVARTIS, MENARINI SPA, GUIDOTTI MALESCI, and grants and personal fees from CHIESI SPA, ASTRA ZENECA, VALEAS, BOEHRINGER INGELHEIM, outside the submitted work. Gianenrico Senna does not have any conflict of interest to report. Pierluigi Paggiaro reports grants and personal fees from AstraZeneca, Chiesi, Novartis and Sanofi, and personal fees from GlaxoSmithKline, Guidotti, Mundipharma, outside the submitted work. Francesco Blasi reports grants and personal fees from Astrazeneca, Chiesi, GSK, Pfizer and Insmed, grants from Bayer, and personal fees from Guidotti, Grifols, Menarini, Mundipharma, Novartis and Zambon, outside the submitted work. Giorgio Walter Canonica reports grants as well as lecture or advisory board fees from: A. Menarini, Alk-Abello, Allergy Therapeutics, AstraZeneca, Boehringer-Ingelheim, Chiesi Farmaceutici, Genentech, Guidotti-Malesci, Glaxo Smith Kline, Hal Allergy, Mylan, Merck, Mundipharma, Novartis, Regeneron, Sanofi-Aventis, Sanofi-Genzyme, StallergenesGreer, UCB pharma, Uriach Pharma, Valeas, ViborPharma.

\section{FUNDING INFORMATION}

SANI is supported through unrestricted grants by AstraZeneca, Glaxo Smith Kline, Novartis \& Sanofi Genzyme.

$$
\begin{array}{r}
\text { Enrico Heffler }{ }^{1,2} \text { iD } \\
\text { Aikaterini Detoraki }^{3} \\
\text { Marco Contoli }{ }^{4} \\
\text { Alberto Papi }^{4} \\
\text { Giovanni Paoletti }^{1,2} \\
\text { Giacomo Malipiero } \\
{ }^{1} \\
\text { Luisa Brussino }^{5} \\
\text { Claudia Crimi }^{6} \\
\text { Daniela Morrone }^{7} \\
\text { Marianna Padovani }^{4} \\
\text { Giuseppe Guida }^{8} \\
\text { rto Giovanni Gerli }{ }^{9} \text { iD } \\
\text { Stefano Centanni }{ }^{10} \\
\text { Gianenrico Senna }{ }^{11} \\
\text { Pierluigi Paggiaro }{ }^{12} \\
\text { Francesco Blasi }{ }^{13,14} \\
\text { gio Walter Canonica } \\
\text { SANI Working Group }
\end{array}
$$$$
\text { Alberto Giovanni Gerli }{ }^{9} \text { iD }
$$$$
\text { Giorgio Walter Canonica }{ }^{1,2}
$$

${ }^{1}$ Personalized Medicine, Asthma and Allergy, Humanitas Clinical and Research Center IRCCS, Rozzano, Italy

${ }^{2}$ Department of Biomedical Sciences, Humanitas University, Pieve Emanuele, Italy

${ }^{3}$ Department of Internal Medicine, Clinical Immunology, Clinical Pathology and Infectious Disease, Azienda Ospedaliera Universitaria Federico II, Napoli, Italy

${ }^{4}$ Department of Morphology, Surgery and Experimental Medicine, University of Ferrara, Ferrara, Italy

${ }^{5}$ Department of Medical Sciences, AO Ordine Mauriziano Umberto I, University of Torino, Torino, Italy

${ }^{6}$ Department of Clinical and Experimental Medicine, University of Catania, Catania, Italy

${ }^{7}$ Severe Asthma Network in Italy (SANI) Infrastructure, Milano, Italy

${ }^{8}$ Allergy and Pneumology Unit, A.O. S. Croce e Carle, Cuneo, Italy

${ }^{9}$ Management Engineering Tourbillon Tech srl, Padova, Italy ${ }^{10}$ Respiratory Unit, Department of Health Sciences, ASST Santi Paolo e Carlo, San Paolo Hospital, University of Milano, Milano, Italy

${ }^{11}$ Asthma Center and Allergy Unit, Verona University Hospital, Verona, Italy

${ }^{12}$ Department of Surgery, Medicine, Molecular Biology and Critical Care, University of Pisa, Pisa, Italy

${ }^{13}$ Internal Medicine Department, Respiratory Unit and Adult Cystic Fibrosis Center, Fondazione IRCCS Cà Granda Ospedale Maggiore Policlinico, Milano, Italy

${ }^{14}$ Department of Pathophysiology and Transplantation, University of Milano, Milano, Italy

Correspondence

Enrico Heffler, Personalized Medicine, Asthma and Allergy, Istituto Clinico Humanitas, Via Alessandro Manzoni 56, 20089 - Rozzano (MI), Italy. Email: enrico.heffler@hunimed.eu

\section{ORCID}

Enrico Heffler (iD https://orcid.org/0000-0002-0492-5663

Alberto Giovanni Gerli iD https://orcid.org/0000-0003-4511-6878

\section{REFERENCES}

1. Civil Protection Department of the Italian Government. http:// opendatadpc.maps.arcgis.com/apps/opsdashboard/index. html\#/b0c68bce2cce478eaac82fe38d4138b1 (Accessed the 18th June 2020).

2. Busse WW, Lemanske RF Jr, Gern JE. Role of viral respiratory infections in asthma and asthma exacerbations. Lancet. 2010;376(9743):826-834

3. Halpin DMG, Faner R, Sibila O, Badia JR, Agusti A. Do chronic respiratory diseases or their treatment affect the risk of SARS-CoV-2 infection? Lancet Respir Med. 2020;8(5):436-438.

4. Chung KF, Wenzel SE, Brozek JL, et al. International ERS/ATS guidelines on definition, evaluation and treatment of severe asthma. Eur Respir J. 2014;43(2):343-373. 
5. Heffler E, Blasi F, Latorre M, et al. The Severe Asthma Network in Italy: findings and perspectives. J Allergy Clin Immunol Pract. 2019;7(5):1462-1468.

6. Jackson DJ, Busse WW, Bacharier LB, et al. Association of respiratory allergy, asthma, and expression of the SARS-CoV-2 receptor ACE2. J Allergy Clin Immunol. 2020;146(1):203-206.

7. Peters MC, Sajuthi S, Deford P, et al. COVID-19 related genes in sputum cells in asthma: relationship to demographic features and corticosteroids. Am J Respir Crit Care Med. 2020;202(1):83-90.

8. Esquivel A, Busse WW, Calatroni A, et al. Effects of omalizumab on rhinovirus infections, illnesses, and exacerbations of asthma. Am J Respir Crit Care Med. 2017;196(8):985-992.

9. Jesenak M, Banovcin P, Diamant Z. COVID-19, chronic inflammatory respiratory diseases and eosinophils - Observations from reported clinical case series. Allergy. 2020;75(7):1819-1822.

\section{SUPPORTING INFORMATION}

Additional supporting information may be found online in the Supporting Information section.

\section{APPENDIX 1}

\section{SANI WORKING GROUP}

M. Bonavia, C. Bucca, M. F. Caiaffa, C. Calabrese, G. Camiciottoli, C. Caruso, M. E. Conte, A. G. Corsico, L. Cosmi, M. T. Costantino, N. Crimi, S. D'Alò, M. D’Amato, S. Del Giacco, A. Farsi, E. Favero, B. M. P. Foschino, G. Guarnieri, S. Lo Cicero, C. Lombardi, L. Macchia, F. Mazza, F. Menzella, M. Milanese, P. Montuschi, M. Montagni, E. Nucera, R. Parente, G. Passalacqua, V. Patella, G. Pelaia, L. Pini, F. Puggioni, L. Ricciardi, F. L. M. Ricciardolo, L. Richeldi, E. Ridolo, G. Rolla, P. Santus, N. Scichilone, P. Solidoro, G. Spadaro, A. Vianello, V. Viviano, M. R. Yacoub, M. C. Zappa 